\title{
2049 Improved detection of myocardial fibrosis using high-resolution, late gadolinium-enhanced cardiovascular magnetic resonance in patients with hypertrophic cardiomyopathy Caitlin J Harrigan*1, Dana C Peters², Martin S Maron³, Barry J Maron4, C Michael Gibson ${ }^{1}$, Warren J Manning ${ }^{1}$ and Evan Appelbaum ${ }^{1}$
}

\author{
Address: ${ }^{1}$ Perfuse CMR Core Lab, Boston, MA, USA, ${ }^{2}$ Beth Israel-Deaconnes Medical Center, Boston, MA, USA, ${ }^{3}$ Tufts-New England Medical \\ Center, Boston, MA, USA and ${ }^{4}$ Minneapolis Heart Institute Foundation, Minneapolis, MN, USA \\ * Corresponding author
}

from I Ith Annual SCMR Scientific Sessions

Los Angeles, CA, USA. I-3 February 2008

Published: 22 October 2008

Journal of Cardiovascular Magnetic Resonance 2008, I0(SuppI I):A3 I8 doi:I0.I I86/I532-429X-I0-SI-A3 I8

This abstract is available from: http://jcmr-online.com/content/I0/SI/A3 I8

(c) 2008 Harrigan et al; licensee BioMed Central Ltd.

\section{Background \\ Myocardial fibrosis as detected by late gadolinium enhanced cardiovascular MR (LGE-CMR) is common in patients with hypertrophic cardiomyopathy (HCM) although its clinical significance is uncertain. Current standard 2D LGE CMR imaging However, a recent post- mortem report demonstrated an isolated foci of papillary muscle scarring which was not detected by conventional 2D LGE-CMR imaging. Three-dimensional high resolu- tion (3D-HR) LGE CMR imaging has the potential for minimizing the partial volume (blood/papillary scar) effect of lower resolution/conventional 2D imaging and may improve our sensitivity for detecting myocardial fibrosis. Therefore, we sought to determine if 3D-HR LGE- CMR has a diagnostic advantage over conventional 2D LGE CMR for the detection of myocardial fibrosis in HCM patients.}

\section{Methods}

Our study compared standard two-dimensional LGE imaging with high resolution, three-dimensional LGE imaging for the detection of myocardial fibrosis in a cohort of patients with hypertrophic cardiomyopathy. Conventional 2D LGE-CMR were acquired 15-20 minutes post injection of $0.2 \mathrm{mmol} / \mathrm{kg}$ GdDTPA, using an ECG-triggered, 40 views per RR, 1 RR between inversions, TI adjusted to null myocardium, fat saturation, $2 \times 2 \times 8$ (skip 2) $\mathrm{mm}$ true spatial resolution, 10-12 short-axis slices, $\mathrm{TR} / \mathrm{TE} / \theta=4.1 \mathrm{~ms} / 1.7 \mathrm{~ms} / 20^{\circ}$ sequence. The $3 \mathrm{D}$ LGE-CMR acquisition immediately followed the $2 \mathrm{D}$ acquisition. Imaging parameters were the same except: respiratory motion compensation with navigator-gating, $\mathrm{TR} / \mathrm{TE} / \theta=5.7 \mathrm{~ms} / 2.8 \mathrm{~ms} / 25^{\circ}, 26$ views per RR, with $1.4 \times$ $1.4 \times 5 \mathrm{~mm}$ true spatial resolution. 2D and 3D LGE-CMR images were obtained in $13 \mathrm{HCM}$ subjects ( $35 \pm 21$ years; $38 \%$ male). All regions of LGE in the left ventricle (LV) and papillary muscles were assessed visually, by 2 independent readers ( $\mathrm{CJH}$ and $\mathrm{EA})$.

\section{Results}

Seven $(54 \%$ ) of 13 HCM subjects (mean LVEF $72 \%$, range 58-82\%; maximal LV wall thickness $20 \mathrm{~mm}$, range 12-50 $\mathrm{mm}$ ) had LV myocardial wall LGE on both 2D and 3D-HR images. On the 3D-HR images, 2/13 (15\%) subjects had papillary muscle enhancement, whereas on the $2 \mathrm{D}$ images, $0 / 13$ subjects had papillary muscle enhancement. Agreement between readers for presence/absence of papillary muscle LGE was $100 \%$.

\section{Conclusion}

These data suggest that 3D-HR LGE CMR imaging can detect myocardial fibrosis not visualized by traditional 2D LGE CMR imaging. Further studies are needed to assess whether high resolution scar imaging should be incorporated into the routine assessment of scar in all HCM patients undergoing CMR examination. 